\title{
STABLE EXTENSIONS BY LINE BUNDLES
}

Let $\mathrm{C}$ be a generic curve of genus g. Denote by $U(n, d)$ the moduli space of stable vector bundles of rank n and degree d on C. Let $E$ be an element of $U(n, d)$. Consider the set of subbundles $E^{\prime}$ of rank $n^{\prime}$ of $E$. Let $E^{\prime \prime}$ be the corresponding quotient and $n^{\prime \prime}$ its rank. We have an exact sequence

$$
(0,1) 0 \rightarrow E^{\prime} \rightarrow E \rightarrow E^{\prime \prime} \rightarrow 0
$$

Define the integer

$$
(0,2) s_{n^{\prime}}(E)=\min \left\{n^{\prime} \operatorname{deg}\left(E^{\prime \prime}\right)-n^{\prime \prime} \operatorname{deg}\left(E^{\prime}\right)\right\}=n^{\prime} d-\operatorname{nmax}\left\{\operatorname{deg}\left(E^{\prime}\right)\right\}
$$

Where the minimum or maximum is taken as $E^{\prime}$ varies in the set of subbundles of $E$ of rank $n^{\prime}$. Then, $s_{n^{\prime}}(E) \equiv n^{\prime} d(n)$. It is known (cf.[N],[M-S]) that $0 \leq s_{1}(E) \leq n^{\prime \prime} n^{\prime} g$. Consider now the following stratification of $U(n, d)$ : denote by

$$
U_{s, n^{\prime}}(n, d)=\left\{E \in U(n, d) \mid s_{n^{\prime}}(E) \leq s\right\}, 0 \leq s \leq n^{\prime} n^{\prime \prime} g
$$

As the function $s_{n^{\prime}}(E)$ is upper semicontinuos in a family of vector bundles, the loci $U_{s}(n, d)$ are closed in $U(n, d)$. The expected dimension of $U_{s}(n, d)$ is given by (see $[\mathrm{L}]$ section 4 )

$$
d_{s, n^{\prime}}=\left(n^{2}-n^{\prime} n^{\prime \prime}\right)(g-1)+s+1
$$

Non-emptiness of $U_{s, n^{\prime}}(n, d)$ will imply in many cases that the expected dimension is in fact the dimension (see [L] p.455). Lange conjectured (cf.[L]) that the loci $U_{s, n^{\prime}}$ should be non-empty for $0 \leq s \leq n^{\prime} n^{\prime \prime}(g-1)$ In this paper prove the conjecture in the case $n^{\prime}=1$ and we shall often drop the subindex 1.

$(\mathbf{0 , 3})$ Theorem. On a generic curve of genus $g \operatorname{dim}_{s, 1}(n, d)=U_{s}(n, d)=d_{s, 1}, 0<s \leq(n-1) g, s \equiv d(n)$.

We are going to prove the result by a degeneration argument. Consider the set of extensions of a rank n-1 vector bundle $\bar{E}$ by a line bundle $L$ so that

$$
\operatorname{deg}(\bar{E})-(n-1) \operatorname{deg}(L)=s
$$

The main point is to show that there is such an extension $E$ that is stable(cf[L], section 4). We do this for a reducible curve made essentially of g elliptic curves forming a chain. We then show that this suffices in order for the result to hold for the generic curve.

The result was known already when $r k E=2(\mathrm{cf}[\mathrm{L}-\mathrm{N}])$ and when

$$
0<\mu\left(E^{\prime \prime}\right)-\mu\left(E^{\prime}\right) \leq(g-1) / \max \left(r k E^{\prime}, r k E^{\prime \prime}\right)
$$

and in a few other special cases ([B-B-R]).

We plan to deal with the general case (arbitrary rank for $E^{\prime}$ ) in the future.

My interest on this question was spurred by a talk given by Barbara Russo at the Europroj 96 meeting. I would like to thank her and the organisers of the conference especially Peter Newstead for giving me the opportunity to attend. I am a member of the Europroj group Vector Bundles on Curves.

\section{The result for the special curve}


In this section $C$ will denote a reducible nodal curve that we construct as follows: Take g generic elliptic curves $C_{1}, \ldots, C_{g}$ with marked points $P_{i}, i=2, \ldots, g ; Q_{i}, i=1, \ldots, g-1, P_{i}, Q_{i} \in C_{i}$. Glue $C_{i}$ to $C_{i+1}$ at $Q_{i}$ and $P_{i+1}$ respectively. We want to prove the equivalent of Theorem $(0,1)$ for this curve.

We shall assume that the reader is familiar with the theory of moduli of stable vector bundles on a reducible curve as developped by Seshadri (cf.[S])(see also [T1,2,3]).

We define $\bar{U}_{s}(n, d)$ as the subset of $U_{s}(n, d)$ of those $E$ such that there is a sequence $(0,1)$ in which the $E^{\prime}, E^{\prime \prime}$ that give the value $s$ are both stable.

$(\mathbf{1}, \mathbf{1})$ Theorem. On the curve $C$ defined above, $\operatorname{dim} \bar{U}_{s, 1}(n, d)=d_{s, 1}, 0<s \leq(n-1) g, s \equiv d(n)$.

We introduce the following notation that we shall use repeatedly. If $E$ is a vector bundle, $E_{P}$ will denote the vector space fiber of $E$ at the point $P$ and $\mathbf{P}\left(E_{P}\right)$ the projective space of lines in this vector space. Also $\mathcal{S}^{k}(E)$ denotes the set of immersed line subbundles of degree $k$ in $E$.

Construct now a vector bundle $E$ on $C$ of degree $d, 0 \leq d<n-1$ in the following way: Denote by $k$ the greatest common divisor of $d$ and $n$. Write $d=k d_{1}, n=k n_{1}$. On the component $C_{1}$ choose a vector bundle $E_{1}$ obtained as the direct sum of $k$ irreducible generic vector bundles of degree $d_{1}$ and rank $n_{1}$. On the components $C_{i}, i \geq 2$, choose a direct sum of $n$ generic line bundles of degree zero. Choose a positive integer $a, 0 \leq a \leq g-1$. Denote by $[x]$ the greatest integer less than or equal to $x$. Define integers $t, k$ as follows if $a \leq g-1-n+d$

$$
(1.2 . a) t=[(g-1-a-n+d) / n], k=g-1-a-n+d-t n
$$

and if $a>g-1-n+d$

$$
(1.2 . b), t=0, k=g-1-a
$$

The gluings will be generic on the first $g-1-a$ nodes. On the last $a$ nodes they will be as described below.

Pick a degree zero line summand of $E_{i}, i=g-a+1 \ldots g$ and glue them together to make a degree zero line subbundle in the chain consisting of the last $a$ curves. Let the gluings be generic otherwise. We shall see the following

$(\mathbf{1 , 3})$ Claim If $a \leq g-1-n+d$, there are line subbundles of degree $-(k+(n-1) t+n-d-1)$ on the union of the first $g-a$ components. Their fibers at $Q_{g-a}$ vary in a subvariety of dimension $k$ of $\mathbf{P}\left(E_{g-a, Q_{g-a}}\right)$. If $a>g-1-n+d$, there are line subbundles of degree $-k$ on the union of the first $g-a$ components. Their fibers at $Q_{g-a}$ vary in a subvariety of dimension $k+d$ of $\mathbf{P}\left(E_{g-a, Q_{g-a}}\right)$.

Glue one of the special directions whose existence is claimed in $(1,3)$ with the direction of the degree zero line subbundle. Let the gluing be generic otherwise.

\section{$(1,4)$ Claim.}

$$
\begin{gathered}
s(E)=d+n(n-d+(n-1) t+k-1), a \leq g-1-n+d \\
s(E)=d+n k, a>g-1-n+d
\end{gathered}
$$

Remark: The set of s obtained in that way cover the whole range of integers $s \equiv d(n), 0 \leq s \leq(n-1) g$.

Let us now count the number of moduli of such families. The choice of $E_{i}$ is the most generic inside one component of the moduli space of vector bundles on $C(\mathrm{cf}[\mathrm{T}] \mathrm{p} .342$, Theorem). The choice of gluings at 
each of the last $a-1$ nodes imposes $n-1$ conditions on the gluing. The gluing at the $g-a^{\text {th }}$ node imposes $n-1-k$ conditions if $a \leq g-1-n+d$ and $n-1-k-d$ conditions if $a>g-1-n+d$. Therefore the number of moduli for such a family is $n^{2}(g-1)+1-(n-1)(a-1)-(n-1-k)$ if $a \leq g-1-n+d$ and $n^{2}(g-1)+1-(n-1)(a-1)-(n-1-k-d)$ if $a>g-1-n+d$. Using the definition $(1,2)$ of $k$, one can check that this number coincides with $d_{s}$.

In order to prove $(1,3)$ and finish the proof of $(1,4)$, we need some preliminary results.

$(\mathbf{1}, \mathbf{5})$ Lemma. Consider generic elliptic curves $C_{1}, \ldots, C_{t}$ with marked points $P_{i}, Q_{i} \in C_{i}$. Form a nodal curve $C$ of genus $t$ by gluing $C_{i}$ to $C_{i+1}$ at $Q_{i}$ and $P_{i+1}$ respectively. Consider a vector bundle $E$ on $C$ such that the restriction $E_{i}$ to $C_{i}$ is a direct sum of $n$ generic line bundles of degree zero and the gluings

$$
\varphi_{i}: E_{i, Q_{i}} \rightarrow E_{i+1, P_{i+1}}
$$

at each node are generic. Consider one-dimensional spaces $V_{1}^{0}$ of $E_{1, P_{1}}$ and $W_{t}^{0}$ of $E_{t, Q_{t}}$. There is a subline bundle $L$ of $E$ of degree at least $-t$ such that $L_{1, P_{1}}=V_{1}^{0}, L_{t, Q_{t}}=W_{t}$ if and only if $t \geq n-1$.

Proof. In order to construct a line subbundle of $E$, one should take a line subbundle $L_{i}$ of each $E_{i}$ such that the fibers at the nodes glue by means of the $\varphi_{i}$.

As $E_{i}$ is a direct sum of $n$ generic line bundles of degree zero, the maximum degree of a line subbundle is zero and there are only a finite number of them. As the gluings are generic, these linesubbundles do not glue with each other.

Recall that $V_{1}^{0}$ is a given one-dimensional subspace of the vector space $E_{1}$. Define the following sets:

$$
\begin{gathered}
A_{j}=\left\{\left(L_{1} \ldots L_{j}, V_{2} \ldots V_{j}, W_{1} \ldots W_{j}\right) \in \mathcal{S}^{-1}\left(E_{1}\right) \times \ldots \times \mathcal{S}^{-1}\left(E_{j}\right) \times \mathbf{P}\left(E_{2, P_{2}}\right) \times \ldots \times \mathbf{P}\left(E_{j, P_{j}}\right) \times\right. \\
\left.\times \mathbf{P}\left(E_{1, Q_{1}}\right) \times \ldots \times \mathbf{P}\left(E_{j, Q_{j}}\right) \mid L_{1, P_{1}}=V_{1}^{0}, L_{i, P_{i}}=V_{i}, i=2 . . j, L_{i, Q_{i}}=W_{i}, i=1 \ldots j, \varphi_{i}\left(W_{i}\right)=V_{i+1}, i=1 . . j-1\right\}
\end{gathered}
$$

(1,6) Claim. $\operatorname{dim} A_{j}=j, 1 \leq j \leq n-1$.

We prove the claim by induction on $\mathrm{j}$.

For every line bundle $L_{1}$ of degree -1 on $C_{1}, h^{0}\left(E_{1} \otimes L_{1}^{-1}\right)=n$. Hence, there is an (n-1)-dimensional projective space of non-zero maps $L_{1} \rightarrow E_{1}$. If we fix one direction $V_{1}^{0} \subset E_{1, P_{1}}$, we obtain a unique such map up to homothety. Therefore, $A_{1}$ is an elliptic curve isomorphic to $C_{1}$.

Assume now the result for $j-1$ and prove it for $j$. There is a natural forgetful map $A_{j} \rightarrow A_{j-1}$. The fiber over a generic point $\left(L_{1} \ldots L_{j-1}, V_{2} \ldots V_{j-1}, W_{1} \ldots W_{j-1}\right)$ is given by

$$
\left\{\left(L_{j}, V_{j}, W_{j}\right) \mid V_{j}=L_{j, P_{j}}=\varphi_{j-1}\left(W_{j-1}\right), W_{j}=L_{j, Q_{j}}\right\} \cong\left\{L_{j} \mid \varphi_{j-1}\left(W_{j-1}\right)=L_{j, P_{j}}\right\}
$$

As in the case of $A_{1}$, one can see that this fiber is an elliptic curve isomorphic to $C_{j}$. Therefore $d i m A_{j}=$ $\operatorname{dim} A_{j-1}+1=j$. This proves $(1,6)$.

$(\mathbf{1}, 7)$ Claim. Denote by $\pi_{i}$ projection from $A_{i}$ on the $i^{\text {th }}$ term. Then $\operatorname{dim} \pi_{3 j-1}\left(A_{j}\right)=j$.

Again, we prove the claim by induction on $\mathrm{j}$. For $\mathrm{j}=1$, we need to see that if $L_{1}, L_{1}^{\prime}$ are different generic vector bundles of degree -1 on $C_{1}$ immersed in $E_{1}$ so that $L_{1, P_{1}}=V_{1}^{0}=L_{1, P_{1}}^{\prime}$, then $L_{1, Q_{1}} \neq L_{1, Q_{1}}^{\prime}$. In fact, as $Q_{1}$ is generic, the opposite would mean that $L_{1, Q}=L_{1, Q}^{\prime}$ for all points $Q \in C_{1}$. Hence the two line subbundles would coincide as bundles immersed in $E_{1}$. As they have the same degree, they should in fact be equal. 
Assume now the result true up to $j-1$ and let us prove it for $j$. As $\operatorname{dim}\left(A_{j}\right)=j$, if $\operatorname{dim}\left(\pi_{3 j-1}\right)^{-1}\left(A_{j}\right)<j$ then the fibers of $\pi_{3 j-1}$ are positive dimensional.

Fix $W_{j} \subset E_{Q_{j}}$. For each $L_{j}$, there is one immersion in $E_{j}$ such that $L_{j, Q_{j}}=W_{j}$. If $\operatorname{dim} \pi_{3 j-1}\left(A_{j}\right) \leq j-2$, then $\operatorname{dim}\left(\pi_{j} \times \pi_{3 j-1}\right)\left(A_{j}\right) \leq j-1=\operatorname{dim}_{3(j-1)-1}\left(A_{j-1}\right)=\operatorname{dim}_{2 j-1}\left(A_{j}\right)$ where the last equality comes from the fact that $W_{j-1}$ determines $V_{j}$ uniquely . This would imply that for a generic point $V_{j} \in \mathbf{P}\left(E_{j, P_{j}}\right)$, there is only a finite number of line bundles $L_{j}$ with $L_{j, P_{j}}=V_{j}$ and this is false. Hence, if the fibers are positive dimensional, they are in fact one-dimensional. Choose an irreducible component $K$ of the fiber. Then $\pi_{j}$ projects it onto $\operatorname{Pic}^{-1}\left(C_{j}\right) \cong C_{j}$. Also, at least one (say $\pi_{k}$ ) of the projections $\pi_{i}, i=1 \ldots j-1$, is not constant. This comes from the fact that $L_{i}$ determines both $V_{i}$ and $W_{i}$ for each i. Denote still with $\pi_{k}, \pi_{j}$ the restrictions of the projections to $K$. Choose any base point in $C_{j}$. Then addition of points of $C_{j}$ is well defined. We define now a non-constant map

$$
\begin{array}{ccc}
C_{k} & \rightarrow & C_{j} \\
P & \rightarrow & \sum_{Q \in \pi_{k}^{-1}(P)} Q
\end{array}
$$

Hence, $C_{k}, C_{j}$ are isogenous. This contradicts the generic choice of the curves and finishes the proof of (1.4).

With the notations introduced so far, Lemma $(1,5)$ amounts to saying that $\pi_{3 j-1}\left(A_{j}\right)$ is a dominant map if and only if $j \geq n-1$. This follows immediately from $(1,7)$.

$(1,8)$ Lemma. Let all the data be as in $(1.4)$ except that now $E_{1}$ is the direct sum of $k$ irreducible generic vector bundles of degree $d_{1}$ and rank $n_{1}$. There is a line subbundle $L$ contained in $E$ with $\operatorname{deg} L \geq-(t-1)$ such that $L_{Q_{t}}=W_{t}^{0}$ if and only if $t \geq n-d$.

Proof of (1.8):A sublinebundle of $E_{1}$ has degree at most 0 . Moreover, given any line bundle $L_{1} \in$ $\mathbf{P i c}^{0}\left(C_{1}\right), h^{0}\left(E_{1} \otimes L_{1}^{-1}\right)=d$. Therefore there is a projective space $\mathbf{P}^{d-1}$ of immersions $L_{1} \rightarrow E_{1}$.

Consider the set

$$
\begin{aligned}
& \bar{A}_{j}=\left\{\left(L_{1} \ldots L_{j}, V_{2} \ldots V_{j}, W_{1} \ldots W_{j}\right) \in \mathcal{S}^{0}\left(E_{1}\right) \times \mathcal{S}^{-1}\left(E_{2}\right) \times \ldots \times \mathcal{S}^{-1}\left(E_{j}\right) \times \mathbf{P}\left(E_{2, P_{2}}\right) \times \ldots \times \mathbf{P}\left(E_{j, P_{j}}\right) \times\right. \\
& \left.\quad \times \mathbf{P}\left(E_{1, Q_{1}}\right) \times \ldots \times \mathbf{P}\left(E_{j, Q_{j}}\right) \mid L_{i, P_{i}}=V_{i}, i=2 . . j, L_{i, Q_{i}}=W_{i}, i=1 \ldots j, \varphi_{i}\left(W_{i}\right)=V_{i+1}, i=1 . . j-1\right\}
\end{aligned}
$$

$\mathbf{( 1 , 9 )}$ Claim. The dimension $\operatorname{dim} \bar{A}_{1}=d=\operatorname{dim} \pi_{2}\left(\bar{A}_{1}\right)$.

Proof of the claim: There is a natural map $A_{1} \rightarrow \mathbf{P i c}^{0}\left(C_{1}\right)$ that is surjective, the fibers being projective spaces of dimension $d-1$. From this, one can compute the dimension of $A_{1}$.

We need to show now that the generic projection has finite fibers. To this end we shall need the following result

$(1,10)$ Claim. We can specialise $E_{1}$ to a direct sum of d line bundles of degree one and n-d line bundles of degree zero.

Proof of $(1,10)$. It is enough to see that an irreducible line bundle of degree $d_{1}$ and rank $n_{1}$ can be specialised to a direct sum of a line bundle $L$ of degree zero and an irreducible vector bundle $F$ of rank $n_{1}-1$ and degree $d_{1}$. The family of extensions of $F$ by $L$ would do the job.

In order to prove $(1,8)$, it is enough to see that the fibers of the map to $\mathbf{P i c}^{0}\left(C_{1}\right)$ map to different subspaces by $\pi_{2}$. In the special case

$$
E_{1}=L^{1} \oplus \ldots \oplus L^{d} \oplus L^{d+1} \oplus \ldots \oplus L^{n}, \operatorname{deg} L^{i}=1, i=1 \ldots d ; \operatorname{deg} L^{i}=0, i=d+1 \ldots n
$$


take the fiber over $L^{d+1}$. With the canonical coordinates in $\mathbf{P}\left(E_{1, Q_{1}}\right)$ given by the decomposition of $E_{1}$, the projection of this fiber contains the open subset $x_{d+1} \neq 0, x_{i}=0, i=d+2, \ldots n$. On the other hand, the projection of the fiber over a generic $L \neq L^{i}, i=1 \ldots n$ is disjoint with this set. This proves $(1,9)$.

Proof of $(1,8)$ : Define

$$
\begin{gathered}
\bar{B}_{j}=\left\{\left(L_{2} \ldots L_{j}, V_{2} \ldots V_{j}, W_{1} \ldots W_{j}\right) \in \mathcal{S}^{-1}\left(E_{2}\right) \times \ldots \times \mathcal{S}^{-1}\left(E_{j}\right) \times \mathbf{P}\left(E_{2, P_{2}}\right) \times \ldots \times \mathbf{P}\left(E_{j, P_{j}}\right) \times\right. \\
\left.\times \mathbf{P}\left(E_{2, Q_{2}}\right) \times \ldots \times \mathbf{P}\left(E_{j, Q_{j}}\right) \mid L_{i, P_{i}}=V_{i}, L_{i, Q_{i}}=W_{i}, i=2 \ldots j, \varphi_{i}\left(W_{i}\right)=V_{i+1}, i=2 . . j-1, W_{j}=W_{j}^{0}\right\}
\end{gathered}
$$

where $W_{j}^{0}$ is a fixed subspace. By $(1,6)$ (applied to the chain in the reverse order), $\operatorname{dim} \pi_{j}\left(\bar{B}_{j}\right)=j-1$. In order for a line subbundle as in $(1,7)$ to exist, we need $\operatorname{dim} \pi_{j}\left(\bar{B}_{j}\right)+\operatorname{dim} \pi_{2}\left(\bar{A}_{1}\right) \geq \operatorname{dim} \mathbf{P}\left(E_{1, P_{1}}\right)=n-1$. Hence, $j \geq n-1$ as claimed.

(1,11) Corollary. $\operatorname{dim} \bar{A}_{j}=d+j-1=\operatorname{dim} \pi_{3 j-1}\left(\bar{A}_{j}\right), 1 \leq j \leq n-d$.

Proof. We already know the result for $j=1$. The computation of the dimension of $\bar{A}_{j}$ is identical to the proof of $(1,5)$. Hence, $\operatorname{dim} \pi_{3 j-1}\left(\bar{A}_{j}\right) \leq d+j-1$. But if the inequality were strict, as this dimension can only increase in one unit with each $j$, then $\operatorname{dim} \pi_{3(n-d)-1}(\bar{A} n-d)<n-1$. Then, $\pi_{3(n-d)-1}$ would not be surjective. On the other hand, (1.8) implies the surjectivity of this map. This contradiction finishes the proof of $(1,11)$.

We can now finish the proof of $(1,3)$ and $(1,4)$. If $a>g-1-(n-d),(1,2)$ and $(1,3)$ are an immediate consequence of $(1,7)$ and $(1,10)$.

Assume $a \leq g-1-n+d$. We want to see that there is a line subbundle whose degree in the chain of components is as follows

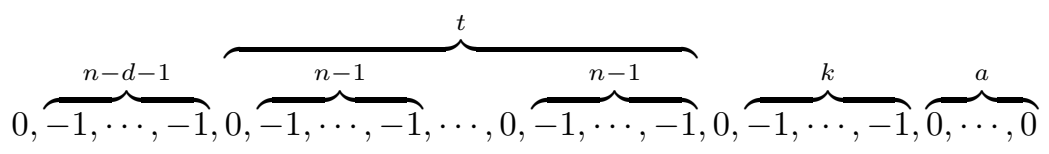

By $(1,8)$, there are such line bundles on the first $n-d$ components and their fibers at $Q_{n-d}$ fill the whole space. One can then glue one of them to one of the degree zero summands of $E_{n-d+1}$. Using $(1,5)$, one can continue this line bundle to the next nt components. Finally by $(1,11)$, one can continue it to the next $k$ components and the fiber at $Q_{g-a}$ moves in the stated dimension.

For a higher degree line bundle to exist, one should replace some of the degree - 1 line subbundles of some $E_{i}$ by degree 0 line subbundles. From $(1,5)$ and $(1,8)$, this is impossible. In order to complete the proof of $(1,1)$, it remains to show that all bundles that appear are (semi)stable for suitably chosen polarisations.

$(1,12)$ Lemma. Consider the vectorbundle $E$, the sublinebundle $L$ and the quotient $E / L$ defined above. Up to tensoring the above data with line bundles of suitable degrees, one can choose polarisations that make them semistable and even stable unless $d \mid n, s=0$.

Proof: Notice that the restriction of $E$ (and of course of $L$ ) to any component is semistable. We show now that the same statement holds for $E / L$.

On the components $C_{2}, \ldots, C_{g}, E_{\mid C_{i}}=L_{1} \oplus \ldots \oplus L_{n}$ where each $L_{k}$ has degree zero and they are generic. When $L_{\mid C_{i}}$ is one of the $L_{k}$ with the natural immersion, the result is obvious. When $L_{\mid C_{i}}$ is a degree -1 line bundle, its image by the immersion in $E_{\mid C_{i}}$ is not contained in any proper direct sum of the degreee zero line subbundles (as the gluing data are generic). Assume the quotient non-stable. Let $F$ be a subbundle of the quotient of degree $d_{F}$ and rank $n_{F}$ that contradicts semistability (i.e. $d_{F} / n_{F} \geq \mu\left(E_{\mid C_{i}} / L_{\mid C_{i}}\right)=1 /(n-1)$ ). 
The pull-back of $F$ to $E$ is then a subbundle of $E$ of degree $d_{F}-1$ and rank $n_{F}+1$ that contains $L_{\mid C_{i}}$. Therefore, $\pi^{-1}(F)$ is not contained in any proper direct sum of the $L_{i}$. This implies that $\operatorname{deg} \pi^{-1}(F)<0$. So $d_{F} \leq 0$ which contradicts the assumption.

We now prove the similar result for $C_{1}$. We shall write $E$ for $E_{\mid C_{1}}$. Again the result is obvious for $d=0$. So let us assume $0<d<n$. We shall show that the quotient of $E$ by a sublinebundle $L$ of degree zero is indecomposable. Assume the opposite. Then, the quotient is the direct sum of indecomposable bundles $F_{1} \oplus \ldots \oplus F_{k}$. The exact sequence

$$
0 \rightarrow L \rightarrow E \rightarrow F_{1} \oplus \ldots \oplus F_{k} \rightarrow 0
$$

corresponds to an extension class

$$
\delta=\left(\delta_{1}, \ldots, \delta_{k}\right) \in H^{1}\left(F^{*} \otimes L\right)=H^{1}\left(F_{1}^{*} \otimes L\right) \oplus \ldots \oplus H^{1}\left(F_{k}^{*} \otimes L\right)
$$

Each $\delta_{i}$ corresponds to an extension

$$
0 \rightarrow L \rightarrow E_{i} \rightarrow F_{i} \rightarrow 0
$$

and we have an exact commutative diagram

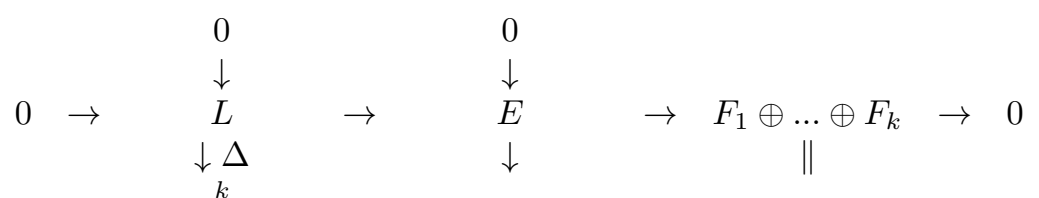

$$
\begin{aligned}
& 0 \rightarrow \overbrace{L \oplus \cdots \oplus L}^{k} \rightarrow E_{1} \oplus \ldots \oplus E_{k} \quad \rightarrow \quad F_{1} \oplus \ldots \oplus F_{k} \quad \rightarrow \quad 0 \\
& \overbrace{L \oplus \cdots \oplus L}^{\substack{k-1 \\
\downarrow}}=\overbrace{L \oplus \cdots \oplus L}^{\substack{\downarrow-1 \\
k \oplus \cdots \oplus}} \\
& \begin{array}{ll}
\downarrow & \downarrow \\
0 & 0
\end{array}
\end{aligned}
$$

where $\Delta$ is the diagonal map.

We first prove that the map from $E$ to any direct summand of any $E_{i}$ cannot be zero: Assume this were the case. Write $E_{i}=E_{i}^{\prime} \oplus E_{i}^{\prime \prime}$ with the map from $E$ to $E_{i}^{\prime}$ being zero. Then, $E_{i}^{\prime}$ injects into $L \oplus \cdots \oplus L$. In particular, it is torsion-free of slope at most 0. Chassing the diagram above, one finds that $L$ surjects onto $E_{i}^{\prime}$ and therefore $E_{i}^{\prime}$ has rank one. It follows that $E_{i}^{\prime}=L, E_{i}^{\prime \prime}=F_{i}$. This contradicts the commutativity of the upper left square.

As $E$ is semistable and $h^{0}\left(E^{*} \otimes E_{i}^{\prime}\right)>0, \mu\left(E_{i}\right)-\mu(E) \geq 0(\operatorname{cf}[\mathrm{A}])$. Equivalently, $n \operatorname{deg}\left(E_{i}\right)-\operatorname{drk}\left(E_{i}\right) \geq 0$. Notice that $\operatorname{deg} E_{i}=\operatorname{deg} F_{i}, r k E_{i}=r k F_{i}+1$. Adding over all inequalities above, we find $n d-d(n-1+k) \geq 0$. So, $1-k \geq 0$ or $k \leq 1$. Hence, $k=1$ and $F$ is irreducible.

Consider now a line bundle $L$ on $C$ of degree $k_{i}$ on the component $C_{i}$. When tensoring a vector bundle $E$ by $L$, the degree of $E$ is modified in $n k_{i}$. Therefore, for a suitable choice of the $k_{i}$, one can bring the degree of the new vector bundle on each component inside any given interval of length $\mathrm{n}$. If the restriction of $E$ to each component is semistable, so is the restriction of the modified vector bundle. By [T1] Theorem (see also [T2]), this is enough to insure stability.

\section{The reduction step}

$(2,1)$ Lemma. Let $C_{0}$ be (a possibly reducible) curve such that $\bar{U}_{s, n^{\prime}}(n, d)\left(C_{0}\right)$ is non-empty and has a component of dimension $d_{s, n^{\prime}}$. Denote by $C_{1}$ the curve obtained from $C_{0}$ by adding chains of rational components between the nodes. Then $\bar{U}_{s, n^{\prime}}(n, d)\left(C_{1}\right)$ is non-empty and has a component of dimension $d_{s, n^{*}}$ 
Proof: Consider the map $\pi: C_{1} \rightarrow C_{0}$ obtained by contracting the rational components. Denote by $E_{1}$ the pull-back by $\pi$ of a vector bundle $E_{0}$ on $C_{0}$. This is trivial (i.e. of the form $\mathcal{O}^{n}$ ) on the additional chains of components. This establishes a one to one correspondence between vector bundles on $C_{0}$ and vector bundles on $C_{1}$ that are trivial on the additional chains of rational components (cf[T3] section 4). This correspondence preserves stability by suitable polarisations (cf [T3] Lemma (1.3)). The subbundles of a trivial vector bundle have degree at most 0 . Also the group of automorphism of a trivial vector bundle is the linear group. This establishes an isomorphism between any two fibers. If the chain glues to the rest at points $Q_{i}, P_{i+1}$ coming from $C_{0}$, this isomorphism coincides with the original gluing on $C_{0}$. One can then see that $s_{n^{\prime}}\left(E_{0}\right)=s_{n^{\prime}}\left(E_{1}\right)$.

Moreover in a neighborhood of $E_{1}$, the elements of the moduli space are trivial on the rational chain (as these are the most generic elements in the moduli space). Hence the dimension of $\bar{U}_{s, n^{\prime}}(n, d)\left(C_{1}\right)$ in a neighborhood of $E_{1}$ coincides with the dimension of $\bar{U}_{s, n^{\prime}}(n, d)\left(C_{0}\right)$ in a neighborhood of $C_{0}$. This completes the proof of $(2,1)$.

$(2,2)$ Proposition. Let $\mathcal{C} \rightarrow \mathcal{S}$ be a family of curves parametrised by a discrete valuation ring. Assume that the generic curve $C_{t}$ is irreducible non-singular while the special curve $C_{0}$ is a curve of compact type (i.e. tree-like). Assume that $C_{0}$ has a vector bundle $E_{0}$ of rank $n$ and degree $d$ that can be written as an extension

$$
0 \rightarrow E_{0}^{\prime} \rightarrow E_{o} \rightarrow E_{0}^{\prime \prime} \rightarrow 0
$$

Assume that (up to tensoring with line bundles on $C_{0}$ of preassigned degree on each component) all data are stable (by polarisations $\left(a_{i}^{\prime}\right),\left(a_{i}\right),\left(a_{i}^{\prime \prime}\right)$ on $C_{0}$ if the curve is reducible). Assume that $s_{n^{\prime}}(E)=s$ and $E^{\prime}$ is a subbundle of rank $n^{\prime}$ with maximal degree. Assume moreover than in a neighborhood of $E_{0}$ The locus of vector bundles with $s_{n^{\prime}}(E)=s$ has dimension $d_{s, n^{\prime}}$. Then in the generic curve, the locus of stable bundles with $s_{1}(E)=s$ is non-empty and has dimension $d_{s, n^{\prime}}$.

Proof: Make a base change if necessary so that $E_{0}^{\prime}, E_{0}, E_{0}^{\prime \prime}$ can be extended to vector bundles on the whole family. One can also assume that the family has enough sections so that moduli spaces of stable bundles on the family exist. One may need to replace the central fiber by a new curve $C_{1}$ which has strings of rational curves inserted between the nodes. By $(2,1)$, the hypothesis on $C_{0}$ hold also for $C_{1}$.

Consider now divisors on $\mathcal{C}$ consisting of sums of components of $C_{1}$ with suitable multiplicity. As $C_{1}$ is of compact type, there are such divisors with any preassigned degree on the components of $C_{1}$. Choose line bundles $\mathcal{L}^{\prime}, \mathcal{L}, \mathcal{L}^{\prime \prime}$, of this form on $\mathcal{C}$. Consider now the moduli spaces $U\left(n^{\prime}, d^{\prime},\left(a_{i}^{\prime}\right)\right), U\left(n^{\prime \prime}, d^{\prime \prime},\left(a_{i}^{\prime \prime}\right)\right)$. The elements of these moduli spaces tensored with $\mathcal{L}^{\prime}$ and $\mathcal{L}^{\prime \prime}$ respectively parametrise vector bundles of the type of $E^{\prime}$ and $E^{\prime \prime}$ that we started with. Then, as in [L] section 4, one can consider families of extensions of this type of bundles. One sees then that the family of stable extensions as stated is either empty or has dimension $d_{s}+\operatorname{dim} S$. As the fiber over $C_{1}$ is non-empty and has dimension $d_{n^{\prime}, s}$, the family of stable extensions projects onto $S$ and the generic fiber has dimension $d_{n^{\prime}, s}$.

Proof of $(0,3)$ : this is a consequence of $(1,1)$ and $(2,2)$.

\section{References}

[A] M.Atiyah, Vector bundles on elliptic curves, Proc.London Math. Soc. (3)7(1957), 414-452.

[B-B-R] E.Ballico, L.Brambila-Paz, B.Russo, Exact sequences of stable vector bundles on projective curves. Math.Nach. To appear.

[L]H.Lange, Zur Klassikation von Regelmannigfaltigkeiten, Math.Ann. 262 (1983), 447-459. 
[L-N] H.Lange, M.S.Narasimhan, Maximal subbundles of rank two vector bundles on curves. Math.Ann. 266 (1983), 55-72.

[M-S] S.Mukai, F.Sakai, Maximal subbundles of vector bundles on a curve, Man.Math. 52 (1985), 251-256.

[N]M.Nagata, On self-intersection number of vector bundles of rank two on a Riemann surface, Nagoya Math. J. 37 (1970), 191-196.

[S] C.S.Seshadri. Fibres vectoriels sur les courbes algebriques. Asterisque 96,(1982).

[T1] M.Teixidor i Bigas, Moduli spaces of (semi)stable bundles on tree- like curves, MathAnn.290 (1991), 341-348.

[T2]-, Moduli spaces of vector bundles on reducible curves, Amer.J.of Math. 117 (1995), 125-139.

[T3]-, Compactifications of moduli spaces of vector bundles on singular curves: two points of view. Preprint.

montserrat teixidor i bigas,

Tufts University, Medford, MA 02155, U.S.A.

Temporary address DPMMS, 16 Mills Lane, Cambridge CB2 1SB, England

teixidor@dpmms.cam.ac.uk or mteixido@pearl.tufts.edu 